\title{
Tumor Mimicking Hepatic Echinococcus Alveolaris with Portal Vein Thrombosis
}

\author{
Fatih Ermişs ${ }^{1}$, Filiz Akyüz ${ }^{1}$, Ahmet Ekmekçi ${ }^{1}$, Memduh Dursun ${ }^{2}$, \\ Mine Güllüoglu ${ }^{3}$ and Kadir Demir ${ }^{1}$
}

Key words: echinococcus alveolaris, portal vein thrombosis

(DOI: 10.2169/internalmedicine.46.0353)

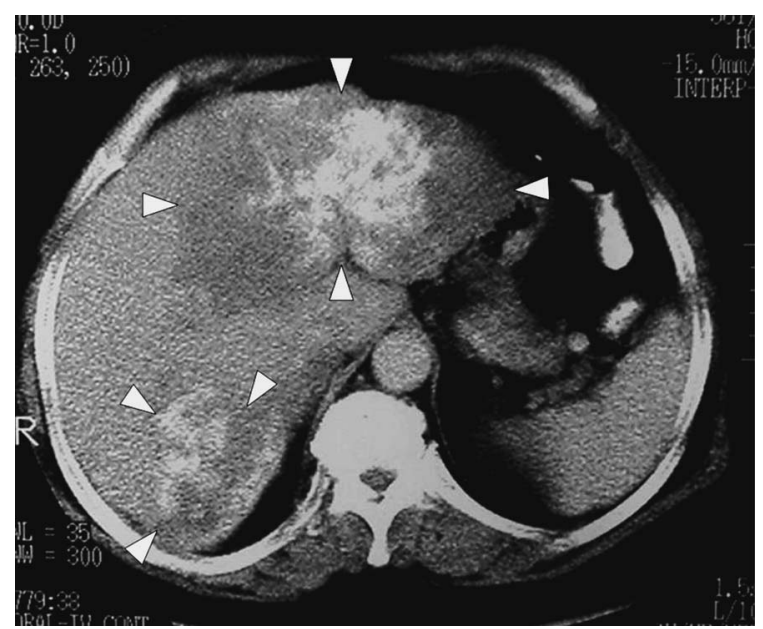

Picture 1. Abdominal CT examination reveals two solid lesions (arrowheads) in the right and left lobe of the liver. The lesions have irregular and ill-defined borders secondary to the infiltrative nature of the lesions. Also these have massive calcifications especially in the left lobe. The lesion located in the left lobe infiltrated the left branch of portal vein.

A 56-year-old woman was referred to our outpatient clinic with a blunt right upper quadrant abdominal pain. An abdominal CT revealed hepatic masses infiltrating both the right and left lobes of the liver and left branch of the portal vein compressing vena cava inferior (Picture 1). Gynecological studies and tumor markers remained negative. Upper gastrointestinal examination showed an extrinsic mass compressing the duodenum, gastrointestinal tumor markers were negative. A tru-cut liver biopsy revealed homogenous faded pink membranous structures in the necrotic fields in HE (hematoxylin and eosin) stained sections and PAS-positive lamellar membranous fragments through the necrotic fields which were patognomonic for E. multilocularis diagnosis.

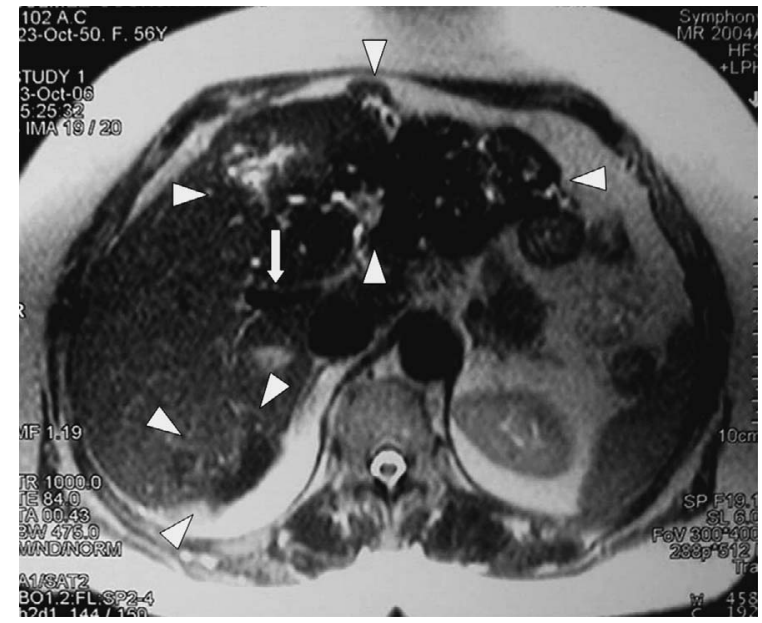

Picture 2. Half-Fourier Acquired Single-Shot Turbo Spin Echo (HASTE) MR axial image shows that the lesions (arrowheads) with ill-defined borders are heterogeneous, secondary to hyperintense cystic regions and hypointense calcifications. Also HASTE MR image shows thrombosis in the portal vein (arrow) not observed in the previous CT examination.

Peripheral blood smear also showed eosinophilia (10\% of $7,600 \mathrm{~mm}^{3}$ leukocytes). Echinococcal indirect hemagglutination test was positive in dilutions up to $1 / 640$. An MRI revealed hepatic hypointense lesions with multiple milimetric hyperintensities within the lesions adjacent to portal vein thrombosis (Picture 2). Based on histology, serology and imaging studies hepatic Echinococcus alveolaris was diagnosed. Since the lesions were widespread, she could not tolerate surgery and was given albendazole treatment. The lesions seemed to be stabilized at the 6-month follow-up.

Human alveolar echinococcus is a potentially fatal, chronic and progressive hepatic disease. After a long asymptomatic period an invasive tumour-like multi-vesiculated and

\footnotetext{
${ }^{1}$ Department of Gastroenterohepatology, Istanbul University, Istanbul Faculty of Medicine, Istanbul, ${ }^{2}$ Department of Radiology, Istanbul University, Istanbul Faculty of Medicine, Istanbul and ${ }^{3}$ Department of Pathology, Istanbul University, Istanbul Faculty of Medicine, Istanbul Received for publication May 29, 2007; Accepted for publication July 19, 2007 Correspondence to Dr. Fatih Ermis, fatihermis2@hotmail.com
} 
exogenously budding lesion develops. There is a risk of metastases in chronic advanced disease; secondary lesions are usually seen in lungs and brain (1). However, portal vein thrombosis in echinococcus infection is unusual. A few cases have been previously reported in the literature $(2,3)$. Here, we presented a rare case diagnosed Echinococcus alveolaris with portal vein thrombosis.

\section{References}

1. Craig P. Echinococcus multilocularis. Curr Opin Infect Dis 16: 437-444, 2003

2. Kayacetin E, Hidayetoglu T. Hydatid cyst of the liver causing a cavernous transformation in the portal vein and complicated by intrabiliary and intraperitoneal rupture. J Gastroenterol Hepatol 19: 1220-1224, 2004.
3. Gil-Egea MJ, Alameda F, Girvent M, Riera R, Sitges-Serra A. Hydatid cyst in the hepatic hilum causing a cavernous transformation in the portal vein. J Gastroenterol Hepatol 21: 227-229, 1998.

(C) 2007 The Japanese Society of Internal Medicine http://www.naika.or.jp/imindex.html 